\title{
Vascular complication following total hip replacement - A nightmare for Arthroplasty surgeon
}

\begin{abstract}
Iatrogenic vascular injuries during total hip arthroplasty (THA) are rare but have serious consequences. The early diagnosis of vascular injuries is often difficult as the signs and symptoms are not specific. The average frequency is between $0.16 \%$ and $0.25 \%$. Various mechanisms described in literature for causing iatrogenic vascular insult are injuries by retractors, mechanical stress, laceration, thrombotic occlusion and formation of false aneurysm. Such complications better be prevented or efficiently treated by thorough preoperative evaluation and vigilant post-operative examination. A case of common femoral artery thrombosis following THA is presented with emphasis on difficult aspect of diagnosis and management.
\end{abstract}

Keywords: iatrogenic, vascular injuries, total hip arthroplasty (THA), common femoral artery, thrombosis
Volume II Issue 5 - 2019

\author{
Yasir Salam Siddiqui,' Sayyed Ehtesham \\ Hussain Naqvi, ${ }^{2}$ Mohd Khalid A Sherwani,' \\ Arshad Ahmad' \\ 'Department of Orthopaedic Surgery, J. N. Medical College, \\ Aligarh Muslim University, India \\ ${ }^{2}$ Department of CTVS, J. N. Medical College,Aligarh Muslim \\ University, India
} Correspondence: Ysir Salc Siddic Ji, Assistant professor, Aligarh Muslim University, Aligarh, Uttar Pradesh, India,Tel +919837343400, Email yassu98@gmail.com

Received: September 12, 2019 | Published: October 28, 2019

\section{Introduction}

Over the years, progressive advancements in the field of arthroplasty have made the total hip arthroplasty procedure relatively safe and well accepted for non salvageable hips. Nevertheless iatrogenic vascular injuries during total hip arthroplasty are rare but have serious consequences on the limb survival and function..$^{1-4}$ The early diagnosis of vascular injuries is often difficult as the signs and symptoms are not specific and moreover these subtle signs are masked by effect of epidural or spinal anaesthesia in immediate post-operative period. The average frequency is between $0.16 \%$ and $0.25 \%$. Various mechanisms described in literature for causing iatrogenic vascular insult are injuries by retractors, mechanical stress, laceration, thrombotic occlusion and formation of false aneurysm. ${ }^{1,2,5-7}$ Such complications better be prevented or efficiently treated by thorough pre-operative evaluation and vigilant post-operative examination. Salama $\mathrm{R}$ et al. ${ }^{8}$ is credited for reporting the first case of an arterial injury during THA. The most commonly affected vessels are the external iliac artery and the common femoral artery. A case of common femoral artery thrombosis following THA is presented with emphasis on difficult aspect of diagnosis and management. An attempt is also made to review the literature on possible mechanisms, risk factors, diagnosis and management of iatrogenic vascular injuries during THA.

\section{Case study}

Case is presented here after taking informed consent from the patient. A 30 year old male with diagnosis of ankylosing spondylitis was admitted for management of end stage arthritis of his right hip (Figure 1). There was no other associated co-morbidity, except for ankylosing spondylitis. However, patient was a chronic smoker. After careful pre-operative assessment, patient was planned for THA. Uncemented THA was performed through anterolateral approach, under general anaesthesia. During operation everything went smoothly including exposure of the hip joint, dislocation of hip, placement of femoral and acetabular components. During the surgery adequate haemostasis was achieved and final closure was done following checking the stability of implanted hip. Patient was extubated and shifted to post-operative recovery room. Immediately after operation patient started screaming with pain in the operated extremity. As described by the patient, he was experiencing altered sensations in his right lower extremity saying that his extremity is feeling very heavy. Examination revealed no movement of ankle or toes. Posterior tibial, dorsalis pedis and even the femoral and popliteal pulses were not palpable. There was no progressive swelling or oozing of blood from the surgical site. There was no activity detected by pulse oxymeter in right lower extremity. Initially there was no appreciable temperature difference between the two extremities. In vain we gently flexed and extended the extremity thinking that the vessels might be in spasm. However, there was no improvement. Moreover the temperature difference became obvious. Hence, a diagnosis of acute post-operative right lower extremity ischaemia was made. Immediately vascular surgeons help was asked for. CT angiogram was done which showed thrombotic occlusion of common femoral artery (Figure 2). Emergency exploration of the femoral artery was done, which revealed thrombotic occlusion. Recanalization was carried out using Forgarty's catheter. Good blood flow was established on removal of the thrombus. Timely intervention by the vascular surgeons restored the circulation and distal pulses. In post-operative period ischaemic pain of the patient was relieved and the movements of the extremity returned after 10 hours (Figure 3 ). The patient was started on anticoagulants therapy for a course of 3 weeks. Rest of the post-operative period was uneventful. Patient was advised to stop smoking. At the latest follow-up about one and half year of the procedure, patient was up and about with no residual neurovascular deficit (Figure 4). 


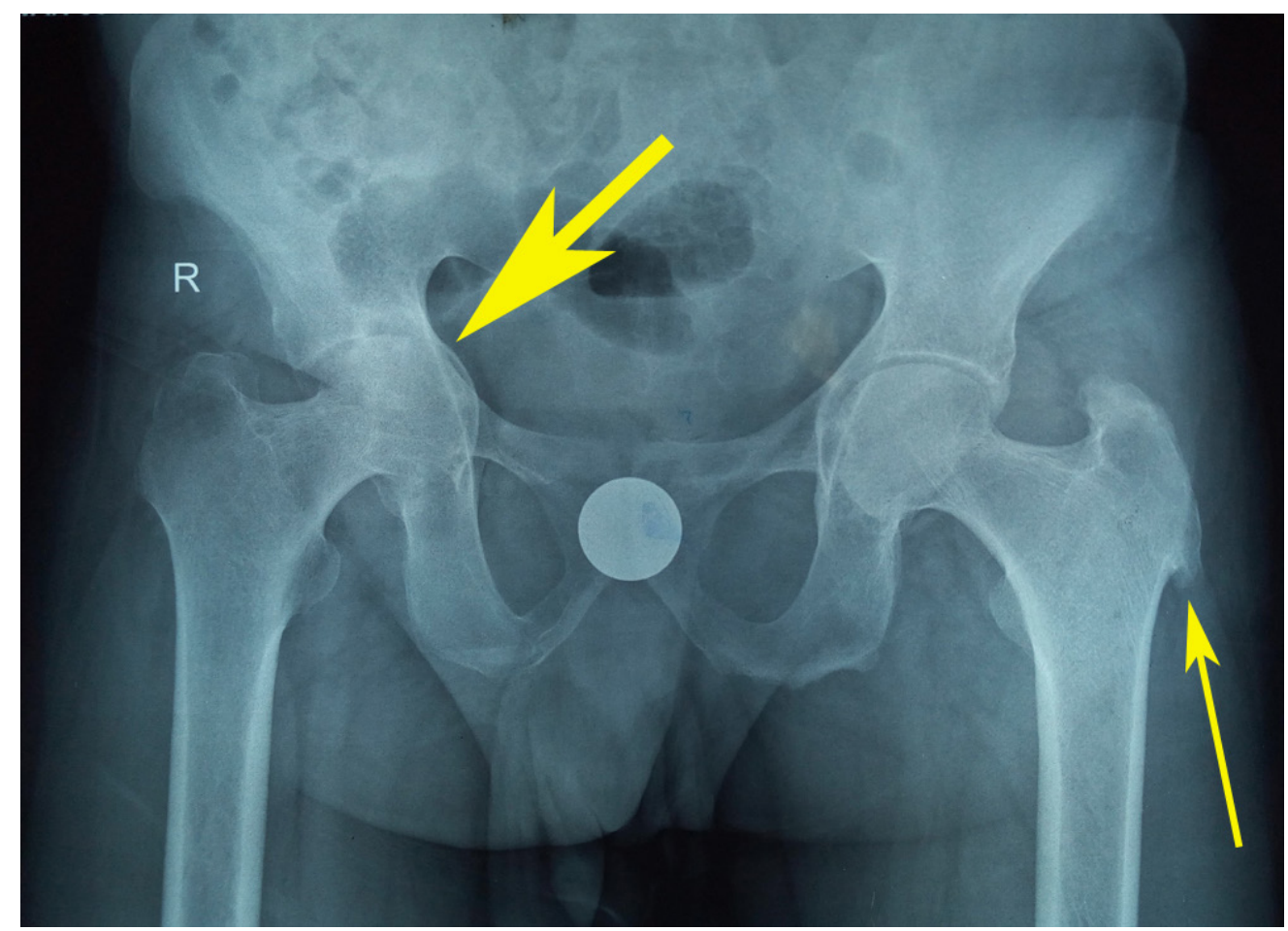

Figure I Pre-operative antero-posterior radiograph of pelvis including both hips of patient showing reduction in joint space on right hip (thick arrow), enthesopathy (thin arrow) with bilateral Sacro-iliac joint ankylosis.

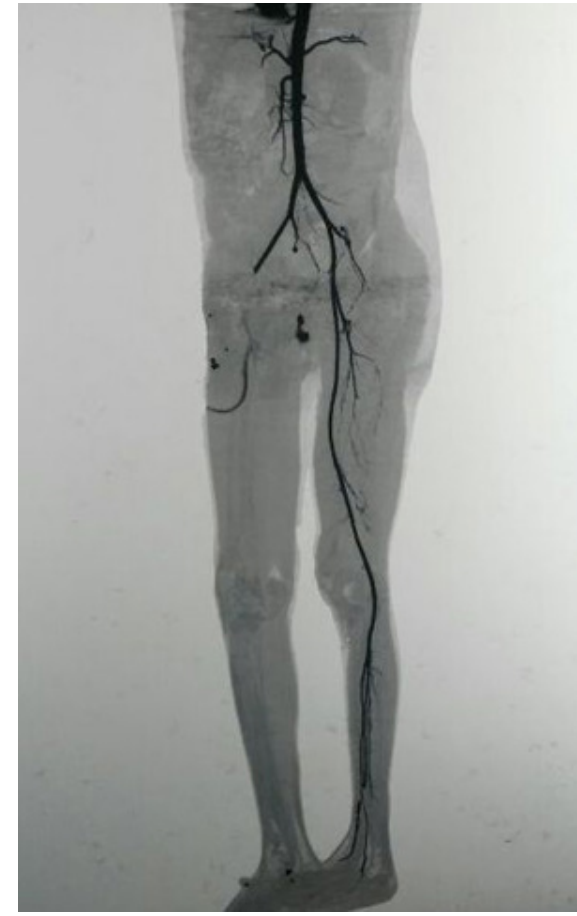

(A)

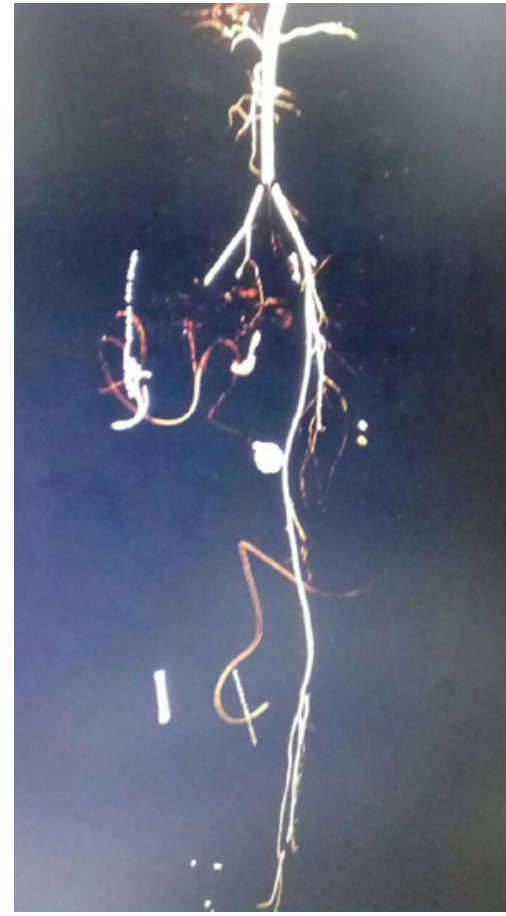

(B)

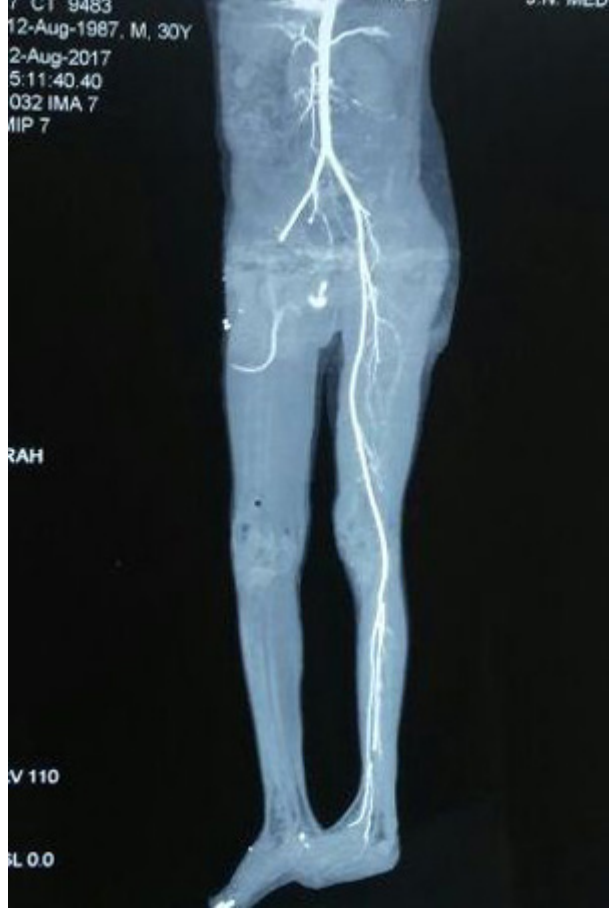

(C)

Figure $2(\mathbf{A}, \mathbf{B}, \mathbf{C})$ Immediate post-operative CT Angiogram showing complete occlusion of right common femoral artery. 


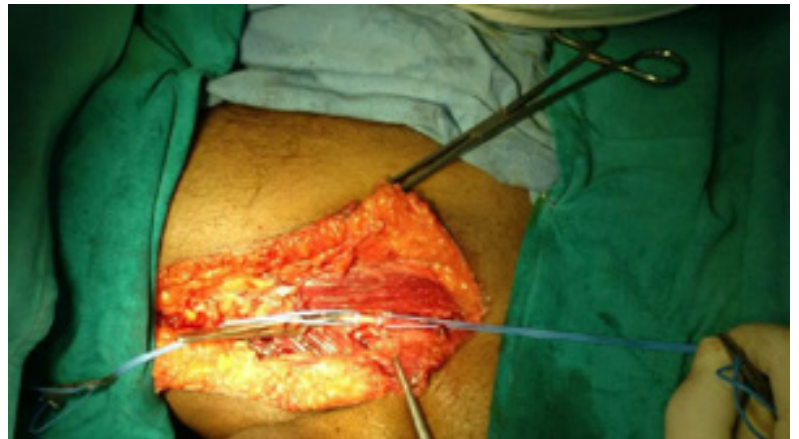

Figure 3 Per-operative photograph showing exploration of right femoral artery.

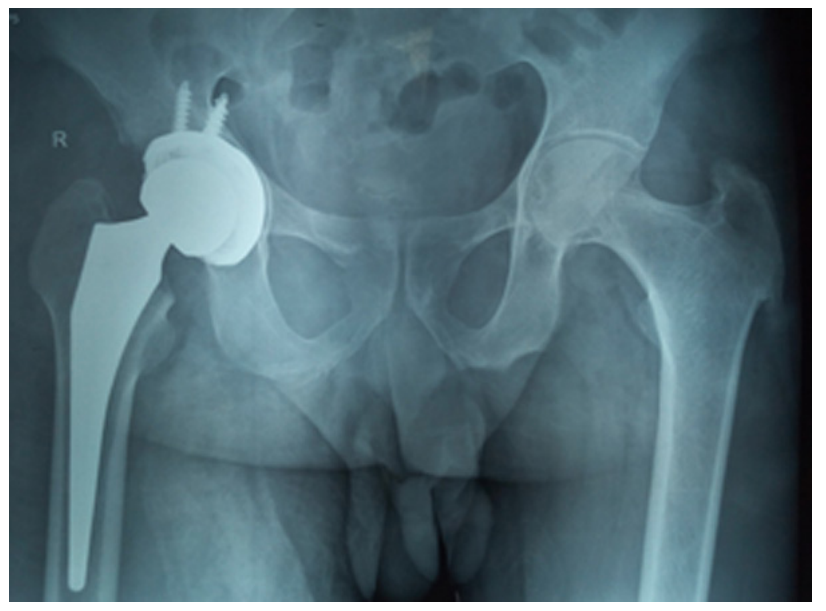

Figure 4 Post-operative antero-posterior radiograph of pelvis including both hips of patient showing uncemented total hip prosthesis.

\section{Discussion}

Iatrogenic vascular injuries during total hip arthroplasty are rare with an average frequency between $0.16 \%$ and $0.25 \%$ as reported by Nachbur B et al. ${ }^{6}$ Others studies have estimated the risk of iatrogenic vascular injury during primary THA to be approximately $0.09 \%{ }^{3,9}$ Of late, Abularrage et al. ${ }^{10}$ reported an incidence of $0.04 \%$ in primary THA increasing to $0.19 \%$ in revision arthroplasty cases. These figures encompassed a wide variety of arterial complications resulting in lower extremity ischaemia, pseudo-aneurysm formation, haemorrhage and arterial transection., ${ }^{3,6}$ Clinical presentation may be delayed, as in the case of pseudo-aneurysms and arterio-venous fistulas ${ }^{6,7}$ or immediately as acute post-operative ischaemia due to traumatic arterial tearing, transection and thrombus formation. ${ }^{6,10}$ Nachbur B et al. ${ }^{6}$ reported two cases of pseudo-aneurysms formation in a series of 15 iatrogenic vascular complications encountered in hip surgery. Predisposing risk factors for development of iatrogenic vascular injuries during THA includes revision surgery, alcoholism, smoking, arteriopathy, vascular bypass, peripheral vascular disease, bone anomalies, anatomic abnormalities and acetabulum or pelvic fractures. ${ }^{1,5,10}$ However, acetabular revision remained the most serious predisposing risk factor. ${ }^{5}$ Meanwhile, Calligaro et al. ${ }^{3}$ did not find any significant difference in primary and revision surgeries, however they reported a rate of acute arterial complications six times higher in total knee arthroplasty than in THA. Duparc ${ }^{1}$ divided the mechanisms of development of iatrogenic vascular injuries into three groups namely, trauma resulting from elongation and torsion by dislocation and reduction manoeuvres, persistent pressure by the tip of Hohmann retractors, or penetration of vessels commonly encountered in revision surgeries. ${ }^{11}$ Nachbur B et al. ${ }^{6}$ also reported injury to vessels by overextension of atherosclerotic vessels with subsequent tear of intima and thrombus formation. Literature also describes possible mechanisms with usage of cement by increasing the chances of leakage leading to a crush injury of the common femoral vessels as well as the chances of thrombotic occlusion from the exothermic reaction during cement polymerization. ${ }^{1,6,10,12}$ Pinpointing the exact cause of acute post-operative ischaemia requires careful clinical examination and investigations (Doppler study, CT angiography). In delayed presentation, insidious clinical picture makes the early diagnosis difficult. In both situations (acute and delayed cases) the slightest doubt calls for urgent exploration of the vessel. ${ }^{1}$ In the study by Calligaro et al. ${ }^{3} 44 \%$ of iatrogenic vascular injuries in total hip and knee arthroplasty procedures failed to be recognized on the day of the surgery. Early diagnosis and timely intervention is the key to prevent catastrophic consequences. Before contemplating any arthroplasty procedure, at first high-risk patients have to be documented. Any history of vascular surgery mandates the opinion of vascular surgeon before arthroplasty. ${ }^{13}$ Careful selection of approach and avoidance of mechanical strain on vessels in dislocating the hip should be sought for high risk patients. The excessive traction during difficult dislocations or reduction manoeuvres in hip surgery can cause arterial tearing. ${ }^{6,14}$

In our case, vessel wall was intact and there was no forced dislocation or reduction of hip to cause mechanical strain to the vessels. The resultant thrombosis was probably due to the persistent pressure by the tip of Hohmann retractor. The predisposing risk factor for thrombosis was the history of smoking and the associated co-morbid condition was the ankylosing spondylitis. Vascular complications if diagnosed too late or left unnoticed can result in debilitating consequences. We were fortunate enough that our anaesthetist were unable to give spinal anaesthesia. Hence patient was operated under general anaesthesia. As soon as patient was awake, he started complaining of severe pain and altered sensations in his right lower extremity. This acute presentation leads to early diagnosis and subsequent timely intervention to save the extremity of our patient, as timely management of such complications greatly depends on the severity with which they present, as was seen in our patient. ${ }^{11}$ Catheter directed thrombolysis can be carried out within 4 to 6hours of presentation. ${ }^{15}$ However, most thrombi are surgically manageable if operated within 24hours. ${ }^{16}$ In our case, removal of thrombus was performed approximately 3hours following the arthroplasty procedure. Fortunately, the limb of the patient could be salvaged. Vascular complications if diagnosed too late or left unnoticed can result in debilitating consequences. Hence, the necessary precautions must be carried out pre-operatively and post-operatively.

\section{Conclusion}

Iatrogenic vascular injuries are rare in total hip arthroplasty. Thrombosis of the common femoral artery is even more rarer. Signs of limb ischemia must be recognized early and necessary investigations/ procedures should be undertaken. Early diagnosis and timely intervention is crucial for preservation of limb.

\section{Acknowledgments}

None. 


\section{Conflicts of interest}

The authors declare there are no conflicts of interest.

\section{References}

1. Duparc F. Vascular risk of orthopaedic and trauma surgery. Teaching Conference. Paris: Scientific Expansion France, Aise Publisher; 1992;46:63-78.

2. Fruhwirth J, Koch G, Ivanic GM, et al. Vascular lesions in surgery of the hip joint. Unfallchirurg. 1997;100(2):119-123.

3. Calligaro KD, Dougherty MJ, Ryan S, et al. Acute arterial complications associated with total hip and knee arthroplasty. J Vasc Surg. 2003;38(6):1170-1177.

4. Kawasaki Y, Egawa H, Hamada D, et al. Location of intrapelvic vessels around the acetabulum assessed by three-dimensional computed tomographic angiography: prevention of vascular-related complications in total hip arthroplasty. J Orthop Sci. 2010;17(4):397-406.

5. Beguin L, Feugier P, Durand JM, et al. Vascular risk and total hip arthroplasty. Rev Chir Orthop Reparatrice Appar Mot. 2001;87(5):489498.

6. Nachbur B, Meyer RP, Verkkala K, et al. The mechanisms of severe arterial injury of the hip joint. Clin Orthop Relat Res. 1979;141:122133.

7. Bach CM, Steingruber IE, Ogon M, et al. Intrapelvic complications after total hip arthroplasty failure. Am J Surg. 2002;183(1):75-79.

8. Salama R, Stavorovsky MM, Iellin A, et al. Femoral artery injury complicating total hip replacement. Clin Orthop Relat Res. 1972;89:143144.
9. Troutman DA, Dougherty MJ, Spivack AI, et al. Updated strategies to treat acute arterial complications associated with total knee and hip arthroplasty. J Vasc Surg. 2013;58(4):1037-1042.

10. Abularrage CJ, Weiswasser JM, Dezee KJ, et al. Predictors of lower extremity arterial injury after total knee or total hip arthroplasty. J Vasc Surg. 2008;47(4):803-807.

11. Rutherford RB. Clinical staging of acute limb ischemia as the basis for choice of revascularization method: when and how to intervene. Semin Vasc Surg. 2009;22(1):5-9.

12. Reilingh ML, Hartemink KJ, Hoksbergen AWJ, et al. Occlusion of the common femoral artery by cement after total hip arthroplasty: a case report. J Med Case Rep. 2009;3:86.

13. Riouallon G, Zilber S, Allain J. Common femoral artery intimal injury following total hip replacement. Orthop Traumatol Surg Res. 2009;95(2):154-158.

14. Schoenfeld NA, Stuchin SA, Pearl R, et al. The management of vascular injuries associated with total hip arthroplasty. J Vasc Surg. 1990;11(4):549-555.

15. Varun GBS, Muralidhar N, Bharathidasan K. A case report: an acute thrombus in the femoral artery following total hip arthroplasty. J Orthop Case Rep. 2016;6(1):55-57.

16. Verstrate M, Verhaeghe R, Belch JJ, et al. Thrombolysis in the Management of Lower Limb Peripheral Arterial Occlusion- A Consensus Document. J VascInterv Radiology. 2003;7:S337-S349. 